\title{
Flux Corrected Finite Volume Scheme for Preserving Scalar Boundedness in Reacting Large-Eddy Simulations
}

\author{
M. Herrmann and G. Blanquart \\ Center for Turbulence Research, Stanford University, California 94305 \\ and \\ V. Raman* \\ University of Texas at Austin, Austin, Texas 78712
}

\section{DOI: $10.2514 / 1.18235$}

\begin{abstract}
Preserving scalar boundedness is an important prerequisite to performing large-eddy simulations of turbulent reacting flows. A number of popular combustion models use a conserved-scalar, mixture-fraction to parameterize reactions that, by definition, is bound between zero and one. To avoid unphysical clipping, the numerical scheme solving the conserved-scalar transport equation must preserve these bounds, while minimizing the amount of numerical diffusivity. To this end, a flux correction method is presented and applied to the quadratic-upwind biased interpolative convective scheme that ensures preservation of the scalar's physical bounds while retaining the low numerical diffusivity of the original quadratic-upwind biased interpolative convective scheme. It is demonstrated that this bounded quadratic-upwind biased interpolative convective scheme outperforms the third-order weighted essentially nonoscillatory scheme in maintaining spatial accuracy and reducing numerical dissipation errors both in generic test cases as well as direct numerical simulation of canonical flows.
\end{abstract}

\section{Introduction}

$\mathbf{L}$ ARGE-EDDY simulation (LES) has emerged as the next generation simulation tool for handling industrially relevant turbulent reacting flows in complex geometries. Of particular interest is the use of LES for modeling complex combustors used both in power-production and aircraft engines [1,2]. Similarly, the chemical processing industry deals with a variety of turbulent flows that involve interaction of mixing and reaction with the final aim of controlling product selectivity and optimizing yield. As the LES method moves from being an academic tool to a practical simulation strategy, robustness of the LES solvers is a key issue to be answered.

Industrially relevant reacting flows often occur in complex geometries, thus necessitating the use of unstructured grid methods to fully capture all geometric detail. This, in practice, limits finite difference and finite volume schemes for the scalar transport equation to schemes with a small, compact stencil, typically schemes with spatial order two or three. Whereas higher-order finite difference and finite volume schemes on structured grids have been devised [3], these are difficult to apply to unstructured grids and thus are not commonly used in industrial applications involving complex geometries.

Industrially relevant reacting flows can cover a range of Mach numbers, from the low-Mach number limit found in stationary burners and aircraft gas-turbine engines, to the fully compressible limit found in SCRAM jets and rocket engines. The present paper focuses on the low-Mach number limit, although the presented methods can be applied in the weakly compressible and fully compressible limit as well.

In low-Mach number combustion, the staggered representation of the primary variables combined with an energy conserving scheme

Presented as Paper 1282 at the 43rd AIAA Aerospace Sciences Meeting and Exhibit, Reno, NV, 10-13 January 2005; received 15 June 2005; revision received 21 August 2006; accepted for publication 28 August 2006. Copyright $\odot 2006$ by the American Institute of Aeronautics and Astronautics, Inc. All rights reserved. Copies of this paper may be made for personal or internal use, on condition that the copier pay the $\$ 10.00$ per-copy fee to the Copyright Clearance Center, Inc., 222 Rosewood Drive, Danvers, MA 01923; include the code $\$ 10.00$ in correspondence with the CCC.

*Department of Aerospace Engineering and Engineering Mechanics. for the momentum equations has been shown to be a stable methodology for a wide variety of flows [4]. In spite of the vast advancement in solving the momentum equations, the scalar transport equations that represent the species distribution inside the geometry, and are thus key to predicting combustor performance, have not been studied in detail. In the present study, we focus on the simulation of a conserved-scalar, namely mixture-fraction. Many combustion models use mixture-fraction to parameterize all species composition [5]. By definition, the solution to the mixture-fraction transport equation should always be in the range $[0,1]$.

Numerical schemes for scalar transport equations are challenging from the viewpoint of accuracy. The advection equation solved using central difference based schemes can lead to significant oscillations near discontinuities [6]. These oscillations, especially if they lead to violation of the physical bounds of the scalar, can significantly decrease the accuracy of a reacting flow simulation. To counter this problem, explicit schemes use an upwind bias that reduces numerical oscillations. As can be expected, this upwind bias also reduces the numerical accuracy of the scheme and leads to artificial diffusion. In spite of this drawback, such schemes are widely used due to their numerical robustness. Despite reducing the undesired oscillations, many of these schemes still do not guarantee that a scalar transport equation solved using bounded initial and boundary conditions preserves the boundedness of the scalar. This property is of special importance in LES, because due to the employed implicit filter size, the filtered spatial scalar distributions tend to exhibit jumps from their minimum to their maximum physical value and vice versa.

In finite volume schemes, cell face fluxes have to be calculated. However, not all quantities used in the evaluation of these fluxes are defined at the cell faces. Thus, an interpolation scheme has to be used to calculate these values there. If such interpolations do not impose a constraint on the local scalar bounds, they can lead to oscillations around the local maximum and minimum values of the scalar. One way to impose such a constraint is the flux correction methodology developed by Zalesak [7]. However, these schemes tend to exhibit relatively high numerical diffusivity. In fact, this artificial diffusivity is so large, that it is comparable in size to the physical subgrid turbulent diffusivity in LES.

An alternative approach is the recently proposed weighted essentially nonoscillatory (WENO) schemes that devise a total 
variation bounded (TVB) nonoscillatory scheme for advection-type equations [8]. Though these methods can be extended to higher-order accuracy, the interpolative coefficients need to be reconstructed at each iterative loop. Extensions to complex unstructured grids are not straightforward and could be computationally expensive.

Another popular approach to removing numerical oscillations is to supplement a higher-order centered scheme with an additional viscositylike [9] or filtering [10] operator. Although the sensor threshold for applying these additional operators are generally based on local gradient information, it is conceivable to apply these operators with an alternative sensor based on physical boundary violation, along the lines of the presented bounded scheme.

Whereas other higher-order and flux limiter based schemes are applicable to the problem at hand, they tend to be of higher numerical cost or lower robustness. However, these two factors are key to the industrial application of LES codes. Thus, in this work, a flux correction method to a well-tested numerical scheme for scalars, namely the quadratic-upwind biased interpolative convective scheme (QUICK) [11] is proposed. This flux correction is constructed in such a way that it adds just the right amount of numerical diffusivity at the right locations to ensure that the global physical bounds of the scalars are maintained. This bounded QUICK (BQUICK) scheme virtually retains the low numerical diffusivity of the original QUICK scheme while maintaining the physical bounds of the scalar.

This paper is structured in the following way. First, the BQUICK scheme and its numerical implementation are presented. Then, results obtained with the BQUICK scheme for some generic numerical test cases are presented and compared with the original QUICK scheme, and the third-order finite volume WENO scheme that is of comparable numerical complexity as the QUICK scheme. Following, the performance of the BQUICK scheme in cases representative of industrial reacting LES, namely a temporal and a spatial evolving mixing layer are presented and compared with the two alternative schemes. Finally, conclusions are drawn.

\section{Bounded QUICK Scheme}

For clarity, the discussion in the following is limited to the onedimensional case. Extension to two and three dimensions and unstructured grids, however, is straightforward.

\section{A. BQUICK Scheme}

The goal of the BQUICK scheme is to solve the simple advection equation

$$
\partial_{t} \phi+\partial_{x}(u \phi)=0
$$

with $\phi$ limited by some physical bounds,

$$
\phi_{\min } \leq \phi \leq \phi_{\max }
$$

where $\phi_{\min }$ and $\phi_{\max }$ have to be known either a priori or as a solution of some other quantities.

In reacting flows, Eq. (1) is typically solved by a standard finite volume approach,

$$
\partial_{t} \phi_{i} \Delta x_{i}=(u \phi)_{i-1 / 2}-(u \phi)_{i+1 / 2}
$$

where $\Delta x_{i}$ is the size of the cell $i$. In LES, staggered formulations of velocity and scalar are usually employed, such that $u_{i \pm 1 / 2}$ is known, but $\phi_{i \pm 1 / 2}$ has to be calculated from $\phi_{i}$. Numerous schemes exist to achieve this, however, two popular schemes in LES are the QUICK scheme [11] and the WENO scheme []. Assuming, without loss of generality, $u_{i-1 / 2}>0$, both schemes can be written as

$$
\phi_{i-1 / 2}=\alpha_{0} \phi_{i}+\alpha_{1} \phi_{i-1}+\alpha_{2} \phi_{i-2}
$$

with

$$
\alpha_{0}=1 / 3, \quad \alpha_{1}=5 / 6, \quad \alpha_{2}=-1 / 6
$$

for the QUICK scheme, and

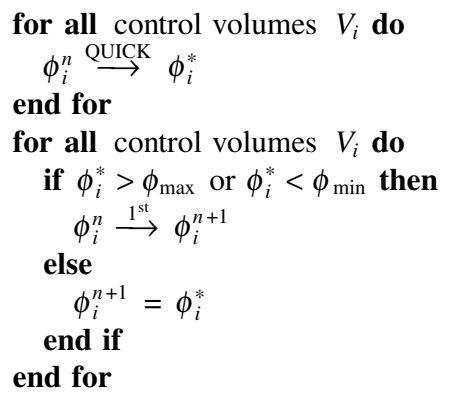

Fig. 1 The BQUICK scheme.

$$
\alpha_{0}=1 / 2 w_{1}, \quad \alpha_{1}=1 / 2 w_{1}+3 / 2 w_{2}, \quad \alpha_{2}=-1 / 2 w_{2}
$$

with

$$
w_{1}=\frac{a_{1}}{a_{1}+a_{2}}, \quad w_{2}=\frac{a_{2}}{a_{1}+a_{2}}
$$

and

$$
a_{1}=\frac{2 / 3}{\left[\epsilon+\left(\phi_{i}-\phi_{i-1}\right)^{2}\right]^{2}}, \quad a_{2}=\frac{1 / 3}{\left[\epsilon+\left(\phi_{i-1}-\phi_{i-2}\right)^{2}\right]^{2}}
$$

for the WENO scheme.

As will be shown later, the QUICK scheme generally exhibits smaller dissipative errors in situations typical of LES than the WENO scheme, but does not ensure scalar boundedness. It is well known, however, that a first-order upwind based interpolation, i.e.,

$$
\alpha_{0}=0, \quad \alpha_{1}=1, \quad \alpha_{2}=0
$$

is unconditionally bounded (within CFL restrictions) but can lead to large dissipative errors. In this work, we use the boundedness property of the first-order upwind scheme to formulate a dual valued stencil for the scalar. Each time-step is split into two substeps that can be constructed as a predictor-corrector algorithm. The predictor step moves the solution from the previous time-step $t^{n}$ to a predicted step $t^{*}$ using the standard QUICK scheme [11]. Then, in cells where the predicted scalar solution at $t^{*}$ is outside the global physical boundaries, Eq. (2), the interpolative scheme is switched to the firstorder upwind scheme. The rest of the domain retains the original QUICK stencil. Using this new stencil and the initial scalar field at $t^{n}$, the corrector step advances the scalar field to the next time level $t^{n+1}$. This modified method is termed a bounded QUICK scheme and is summarized in Fig. 1.

BQUICK in fact follows the methodology of a flux correction scheme [7], but uses a global physical constraint on the scalar instead of a local one. This limits the application of the first-order correction to only those cells that would violate the boundedness property while everywhere else, the lower numerical dissipation of the original QUICK scheme is maintained. In practical LES, time-integration is typically performed by a semi-implicit iterative procedure [12]. In such cases, the preceding predictor-corrector scheme is executed in each subiteration with the initial scalar values determined by the field at the previous subiteration.

In principle, the preceding predictor-corrector algorithm is applicable to any higher-order transport scheme, thus making the corrected scheme obey the physical bounds on the scalar, Eq. (2). However, due to the focus of this paper on industrial applications, we will limit the discussion in the following to the two most commonly used finite volume schemes in unstructured grid methods, the QUICK scheme, its BQUICK variant, and the WENO scheme.

\section{B. Numerical Stability}

Both the QUICK and the first-order upwind scheme are analytically stable as can be seen easily from a simple von Neumann stability analysis. Assuming uniform mesh spacing and uniform positive velocity, the discretization of the QUICK scheme yields 


$$
\partial_{t} \phi_{i}=\frac{u}{6 \Delta x}\left(2 \phi_{i+1}+3 \phi_{i}-6 \phi_{i-1}+\phi_{i-2}\right)
$$

Decomposition in Fourier modes and analysis of the amplification of a given mode of amplitude $\hat{\phi}_{k}$ corresponding to a wave number $k$ yields

$$
\partial_{t} \hat{\phi}_{k}+\alpha \hat{\phi}_{k}=0
$$

where the coefficient $\alpha$ is generally complex. In the case of the QUICK scheme, the real part of $\alpha$ is always positive,

$$
\Re(\alpha)=\frac{u}{3 \Delta x}[\cos (k \Delta x)-1]^{2}>0
$$

thus proving stability of the QUICK scheme, provided proper timeintegration schemes are used. The same analysis can be applied to the first-order scheme. Because both components of the BQUICK scheme are stable, one can have strong confidence that the combination of the two schemes, in the manner proposed, also results in a stable scheme.

\section{Reacting Scalars}

The BQUICK method for preserving scalar boundedness has been discussed in the preceding section for the case of passive scalars. Whereas passive scalars form the basis of several popular combustion models, reactive scalars are also common to turbulent combustion models [5]. Although the following section focuses on three common examples, the scalar variance, the progress variable, and the mass-fractions of chemical species, it can be used as guidelines for applying the BQUICK scheme to other reactive scalars.

It should be pointed out that typically the main source of violating the physical bounds of a reactive scalar is due to errors in the evaluation of the scalar source term. There exist several methods to perform accurate time-integration while preserving the bounds of the scalars even in the case of very stiff chemical source terms [13], and for the purpose of the present section it is assumed that the source term evaluation does preserve the physical boundaries. Then, all violations of the physical bounds are due to the advection of the scalar.

As stated in Eq. (2), the physical bounds of a scalar used in the BQUICK scheme have to be known. Although Eq. (2) is applied in a global manner in the case of passive scalars, this limitation can be dropped for reactive scalars. In the case of scalar variance $\phi^{\prime 2}$, the physical lower bound is zero, whereas the physical upper bound is given by the solution of the scalar equation as $\phi(1-\phi)$. Thus, solving first for $\phi$, determining the local lower and upper physical bound of the scalar variance as $\phi_{\min }^{\prime 2}=0$ and $\phi_{\max }^{\prime 2}=\phi(1-\phi)$, the BQUICK scheme can readily be applied to the scalar variance advection step.

In the case of a progress variable $c$, it can be shown that the evolution of the progress variable is bounded between two functions of the mixture-fraction, $c_{\min }(Z)$ and $c_{\max }(Z)$. Given the solution to the mixture-fraction equation $Z$, these bounds can be calculated locally using pretabulated data and enforced in the BQUICK scheme for advection of the progress variable scalar.

Finally, in the case of mass-fractions, each of the mass-fractions is physically bounded between zero and one. Determining more restrictive physical boundaries is a difficult, if not impossible, task. However, there exists an additional constraint when solving for the mass-fractions of all species, namely that locally

$$
\sum_{i=1}^{N} Y_{i}(x, t)=1
$$

This constraint should be enforced strictly for local conservation of mass. Because the QUICK and the first-order upwind schemes are fully linear, they satisfy by default this conservation criterion. In the BQUICK scheme, if either the physical bounds of zero and one (of one of the mass-fractions) or Eq. (13) is violated in the predictor step, the local first-order update has to be applied to all the mass fraction scalars simultaneously. This ensures that Eq. (13) remains strictly enforced. It should be pointed out that most of the available scalar transport schemes, including the WENO scheme, violate Eq. (13) because the interpolation method is inherently nonlinear and based on each individual scalar.

\section{Numerical Tests}

A series of tests are used to compare the new BQUICK scheme with the original QUICK scheme and the third-order WENO scheme. In the first set, three one-dimensional generic test cases are analyzed, whereas in the second set both spatial and temporal evolving threedimensional mixing layers are studied.

\section{A. One-Dimensional Tests}

To determine the order of accuracy, a sine wave function extending over a domain of $[0,2 \pi]$ is convected using a constant velocity and imposing periodic boundary conditions. Because the sine wave is a smooth function, numerical dispersion errors are minimized. Two different functions are used to test the effect of sharper curves. The first test uses as an initial condition

$$
\phi(x)=\frac{1}{2}[\sin (\pi x)+1]
$$

with $\phi_{\min }=0$ and $\phi_{\max }=1$. The simulations are carried out with increasing resolution up to a grid of 1024 points using a fourth-order Runge-Kutta based time-integration. Using the exact solution, $L_{1}$, $L_{2}$, and $L_{\infty}$ errors in the predictions are computed. Table 1 shows the errors in each simulation after one period for the three different numerical schemes. It is seen from the $L_{2}$ error that the BQUICK and QUICK schemes both show slightly more than second-order accuracy, whereas the WENO scheme shows more than fourth-order accuracy. Note, however, that although the WENO scheme exhibits higher-order convergence rates, the absolute errors are nonetheless

\begin{tabular}{|c|c|c|c|c|c|c|c|}
\hline Method & $\mathrm{N}$ & $L_{\infty}$ error & $L_{\infty}$ order & $L_{1}$ error & $L_{1}$ order & $L_{2}$ error & $L_{2}$ order \\
\hline \multirow[t]{4}{*}{ QUICK } & 128 & $1.07 \mathrm{E}-04$ & - & $6.81 \mathrm{E}-05$ & - & $6.69 \mathrm{E}-06$ & 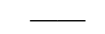 \\
\hline & 256 & $2.56 \mathrm{E}-05$ & 2.06 & $1.63 \mathrm{E}-05$ & 2.06 & $1.13 \mathrm{E}-06$ & 2.56 \\
\hline & 512 & $6.33 E-06$ & 2.02 & $4.03 E-06$ & 2.02 & $1.98 \mathrm{E}-07$ & 2.52 \\
\hline & 1024 & $1.58 \mathrm{E}-06$ & 2.00 & $1.00 \mathrm{E}-06$ & 2.00 & $3.49 \mathrm{E}-08$ & 2.50 \\
\hline \multirow[t]{4}{*}{ BQUICK } & 128 & $1.15 \mathrm{E}-04$ & & $7.27 \mathrm{E}-05$ & & $6.97 \mathrm{E}-06$ & \\
\hline & 256 & $2.85 \mathrm{E}-05$ & 2.01 & $1.75 \mathrm{E}-05$ & 2.06 & $1.18 \mathrm{E}-06$ & 2.57 \\
\hline & 512 & $7.24 \mathrm{E}-06$ & 1.98 & $4.27 \mathrm{E}-06$ & 2.03 & $2.04 \mathrm{E}-07$ & 2.53 \\
\hline & 1024 & $1.92 \mathrm{E}-06$ & 1.92 & $1.05 \mathrm{E}-06$ & 2.03 & $3.56 \mathrm{E}-08$ & 2.52 \\
\hline \multirow[t]{4}{*}{ WENO-3 } & 128 & $7.00 \mathrm{E}-03$ & - & $1.40 \mathrm{E}-03$ & - & $2.10 \mathrm{E}-04$ & \\
\hline & 256 & $1.28 \mathrm{E}-03$ & 2.45 & $1.61 \mathrm{E}-04$ & 3.12 & $2.01 \mathrm{E}-05$ & 3.39 \\
\hline & 512 & $8.94 \mathrm{E}-05$ & 3.84 & $1.16 \mathrm{E}-05$ & 3.80 & $8.84 \mathrm{E}-07$ & 4.51 \\
\hline & 1024 & $3.11 \mathrm{E}-06$ & 4.85 & $1.27 \mathrm{E}-06$ & 3.19 & $4.45 \mathrm{E}-08$ & 4.31 \\
\hline
\end{tabular}
larger than both the QUICK and the BQUICK schemes, even for the finest resolution of 1024 grid cells. Also, the BQUICK scheme shows only marginally increased error levels as compared with the

Table 1 Accuracy for $\phi(x)=\frac{1}{2}[\sin (\pi x)+1]$ 
Table 2 Accuracy for $\phi(x)=\sin ^{4}(\pi x)$

\begin{tabular}{|c|c|c|c|c|c|c|c|}
\hline Method & $\mathrm{N}$ & $L_{\infty}$ error & $L_{\infty}$ order & $L_{1}$ error & $L_{1}$ order & $L_{2}$ error & $L_{2}$ order \\
\hline \multirow[t]{4}{*}{ QUICK } & 128 & $3.55 \mathrm{E}-03$ & - & $1.81 \mathrm{E}-03$ & $\bar{L}$ & $1.83 \mathrm{E}-04$ & 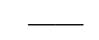 \\
\hline & 256 & $6.54 \mathrm{E}-04$ & 2.44 & $3.29 \mathrm{E}-04$ & 2.46 & $2.38 \mathrm{E}-05$ & 2.95 \\
\hline & 512 & $1.45 \mathrm{E}-04$ & 2.18 & $7.20 \mathrm{E}-05$ & 2.19 & $3.71 \mathrm{E}-06$ & 2.68 \\
\hline & 1024 & $3.49 \mathrm{E}-05$ & 2.05 & $1.73 \mathrm{E}-05$ & 2.06 & $6.32 \mathrm{E}-07$ & 2.55 \\
\hline \multirow{4}{*}{ BQUICK } & 128 & $3.59 \mathrm{E}-03$ & - & $1.77 \mathrm{E}-03$ & & $1.86 \mathrm{E}-04$ & \\
\hline & 256 & $6.51 \mathrm{E}-04$ & 2.47 & $3.27 \mathrm{E}-04$ & 2.43 & $2.42 \mathrm{E}-05$ & 2.94 \\
\hline & 512 & $1.45 \mathrm{E}-04$ & 2.17 & $7.22 \mathrm{E}-05$ & 2.18 & $3.74 \mathrm{E}-06$ & 2.70 \\
\hline & 1024 & $3.49 \mathrm{E}-05$ & 2.05 & $1.74 \mathrm{E}-05$ & 2.05 & $6.34 \mathrm{E}-07$ & 2.56 \\
\hline \multirow{4}{*}{ WENO-3 } & 128 & $6.70 \mathrm{E}-02$ & - & $1.49 \mathrm{E}-02$ & - & $2.02 \mathrm{E}-03$ & \\
\hline & 256 & $2.41 \mathrm{E}-02$ & 1.48 & $3.27 \mathrm{E}-03$ & 2.19 & $4.06 \mathrm{E}-04$ & 2.32 \\
\hline & 512 & $6.35 \mathrm{E}-03$ & 1.92 & $5.15 \mathrm{E}-04$ & 2.67 & $5.73 \mathrm{E}-05$ & 2.83 \\
\hline & 1024 & $9.03 \mathrm{E}-04$ & 2.81 & $5.35 \mathrm{E}-05$ & 3.27 & $4.26 \mathrm{E}-06$ & 3.75 \\
\hline
\end{tabular}

QUICK scheme. This is not surprising because the corrector step can only be active near $\phi_{\min }$ and $\phi_{\max }$ that make up only a small portion of the computational domain.

The second test case uses a function with a sharper profile for the initial conditions,

$$
\phi(x)=\sin ^{4}(\pi x)
$$

again with $\phi_{\min }=0$ and $\phi_{\max }=1$. Table 2 shows the errors as well as the accuracy estimates for the different numerical schemes. The QUICK and BQUICK schemes have retained second-order accuracy for this profile. In fact, both schemes show almost identical results as the previous test case. However, the WENO scheme shows a dramatic reduction in accuracy. The order of the scheme has dropped from over four in the previous case to between 2.5-3.5. Again, the absolute values of the errors are noticeably smaller for the QUICK and the BQUICK schemes as compared with the WENO scheme.

Though the preceding two tests reveal the truncation error, scalar profiles in turbulent flow are rarely smooth. They rather exhibit strong gradients typically directly from the minimum to the maximum physical value and vice versa. To test such a scenario, a canonical 1-D convection problem [14] is used. As initial conditions, a profile consisting of several shapes with sharp gradients are distributed across the domain discretized by 256 equidistant cells. A uniform velocity field is imposed with periodic boundaries. Again, a fourth-order Runge-Kutta based time-integration is used to minimize temporal errors. The simulation is carried out for four rotations where each rotation is defined as the time taken for the initial profile to be convected back to its initial location.

Figure 2 compares the results from the QUICK, BQUICK, and WENO schemes. It can be seen that the QUICK scheme locally exceeds the initial bounds of the scalar. In addition, it exhibits oscillations near regions of sharp gradients. The BQUICK scheme on the other hand maintains the boundedness accurately while also reducing the unphysical oscillations. Compared with the WENO scheme, the BQUICK scheme performs noticeably better at capturing the local extrema of the scalar. This result indicates that the WENO scheme will lead to significantly more undesired damping of the resolved scalar fluctuations in a large-eddy simulation, whereas the BQUICK scheme will maintain these scalar fluctuations better, while retaining the physical bounds of the scalar.

\section{B. Mixing Layer Tests}

The second set of tests consists of an actual multidimensional implementation of the three schemes (QUICK, BQUICK, and WENO) into a LES solver. Two configurations are investigated: a spatially evolving mixing layer using the LES formulation and a temporal mixing layer employing sufficient spatial resolution to be in essence a DNS.

\section{Governing Equations}

The equations solved for the mixing layers correspond to the lowMach number formulation of the Favre averaged Navier-Stokes equations. In the general case of LES of reacting flows, they take the following form:

$$
\partial_{x_{i}} \bar{\rho} \tilde{u}_{i}=0
$$

$$
\partial_{t} \bar{\rho} \tilde{u}_{i}+\partial_{x_{j}} \bar{\rho} \tilde{u}_{i} \tilde{u}_{j}=-\partial_{x_{i}} \bar{p}+\partial_{x_{j}} \bar{\tau}_{i j}
$$

$$
\partial_{t} \bar{\rho} \tilde{Z}+\partial_{x_{i}} \bar{\rho} \tilde{u}_{i} \tilde{Z}=\partial_{x_{i}} \bar{G}_{i}
$$

with

$$
\bar{\tau}_{i j}=\bar{\mu}\left(\partial_{x_{i}} \tilde{u}_{j}+\partial_{x_{j}} \tilde{u}_{i}\right)-\frac{2}{3} \bar{\mu} \delta_{i j} \partial_{x_{k}} \tilde{u}_{k}+\bar{\tau}_{i j}^{\mathrm{SGS}}
$$
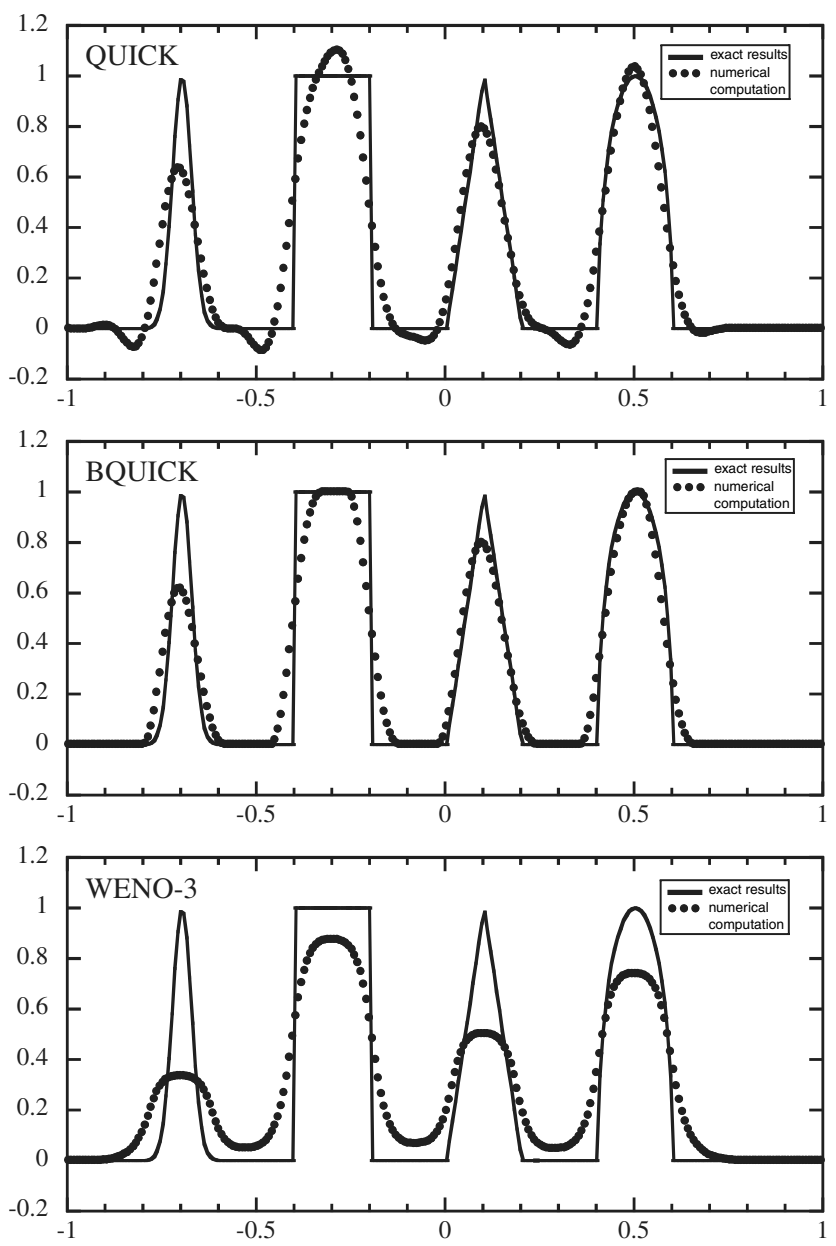

Fig. 2 1-D convection test case. Comparison of numerical schemes after four rotations. 


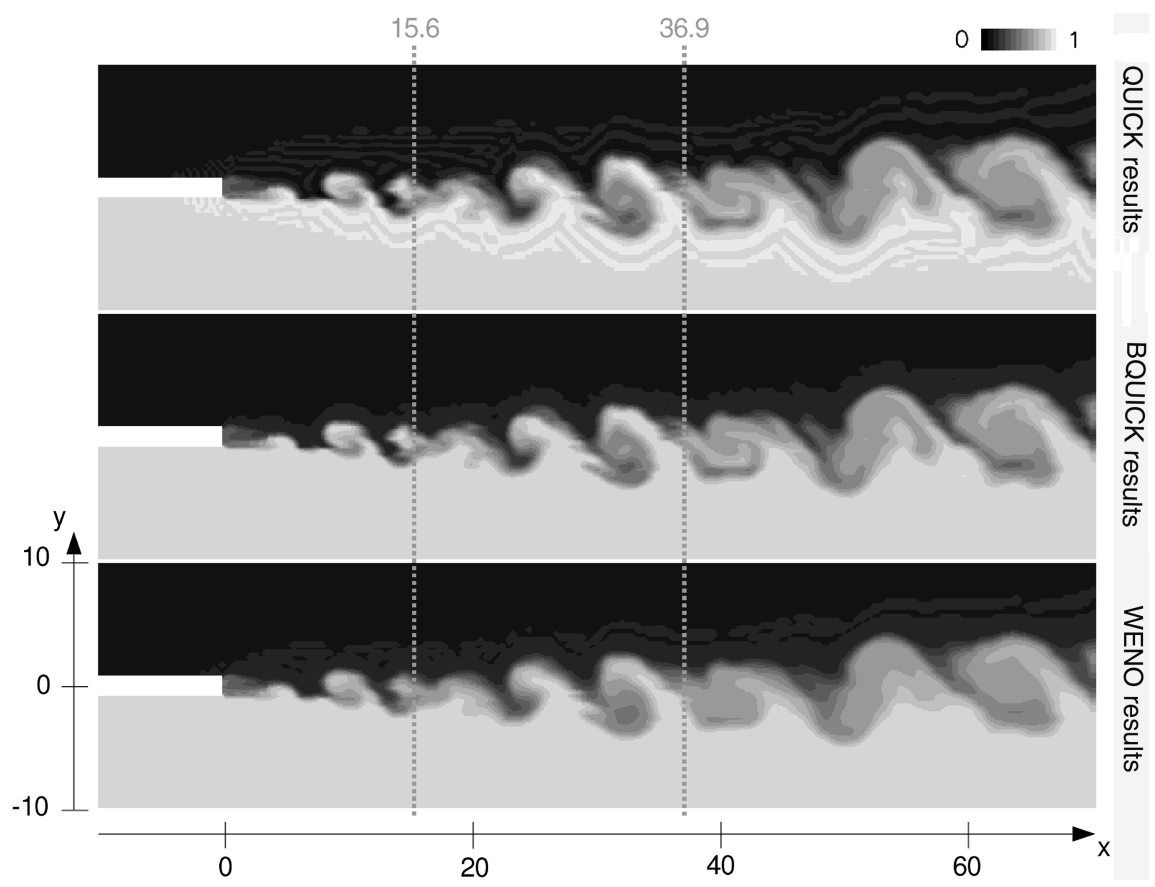

Fig. 3 Two-dimensional profile of the instantaneous mixture-fraction in a mixing layer.

and

$$
\bar{G}_{i}=\bar{D} \partial_{x_{i}} \tilde{Z}+\bar{G}_{i}^{\mathrm{SGS}}
$$

The subgrid scale model used in the spatially evolving mixing layer is a dynamic Smagorinsky model. For the DNS of the temporal mixing layer, the subgrid scale model is switched off, i.e., $\bar{\tau}_{i j}^{\text {SGS }}=0$ and $\bar{G}_{i}^{\text {SGS }}=0$.

The equations for both of these simulations are solved in nondimensional units using a domain decomposition based parallel solver. Further details of the code as well as the subfilter models used (in the case of LES) can be found in [6]

\section{Spatial Mixing Layer}

A splitter plate divides two streams up to a coordinate of $X=0$, from which point on the spatial mixing layer trips and evolves. The inflow bulk velocities of the two streams are set to a ratio of $1: 2.5$ with plug flow profiles and the mixture-fraction is set to zero in the upper stream and one in the lower stream. The domain, including the splitter plate, stretches for 80 units whereas the width is set at 20 units. The Reynolds number based on the higher speed stream velocity and the splitter plate thickness is $R e=20,000$. Figure $\underline{3}$ shows the instantaneous scalar concentration using the three schemes. The QUICK and the WENO scalar fields have been clipped between zero and one, whereas the BQUICK maintains the physical bounds. Even if the WENO is not perfectly bounded, the overshoots and undershoots (of the order of \pm 0.0001 ) are much smaller than those of the QUICK scheme (of the order of \pm 0.1 ). Visually, apart from the stripes due to the clipping procedure, the differences between the QUICK and BQUICK schemes are not noticeable. On the other hand, the WENO scalar field is smoother than the two other schemes. To better understand the effect of the BQUICK scheme, crosswise scalar profiles at $X=15.6$ are plotted in Fig. 4 a. It is seen that BQUICK maintains the same profile as the QUICK scheme away from the bounds of the scalar. However, closer to zero and one, BQUICK adjusts itself to maintain the bounds. The WENO profile is also bounded but it is much smoother than the BQUICK profile and does not show the same scalar gradient.

The lower-order correction for the BQUICK scheme at the bounds in composition space has limited impact on the physical transport. To further substantiate this argument, time-averaged profiles at $X=$ 36.9 of both the mixture-fraction as well as the mixture-fraction variance are plotted in Fig. $\underline{4 b}$. Increased numerical diffusion of the first-order correction in the BQUICK scheme should reduce the variance as compared with the original QUICK scheme. Although a minute decrease in variance value is indeed observed, its magnitude is not substantial because the corrections are applied only in a very

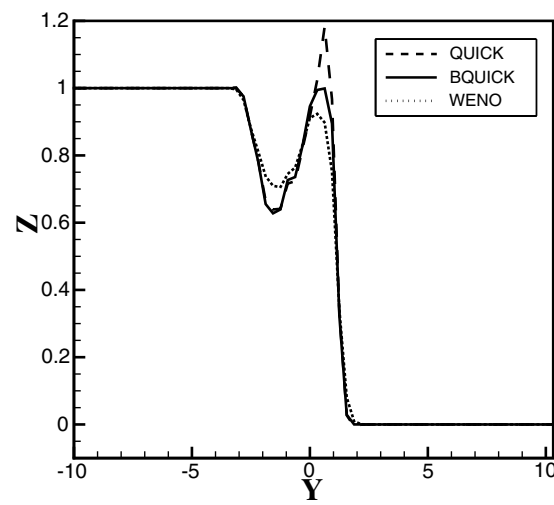

a) Instantaneous, $X=15.6$

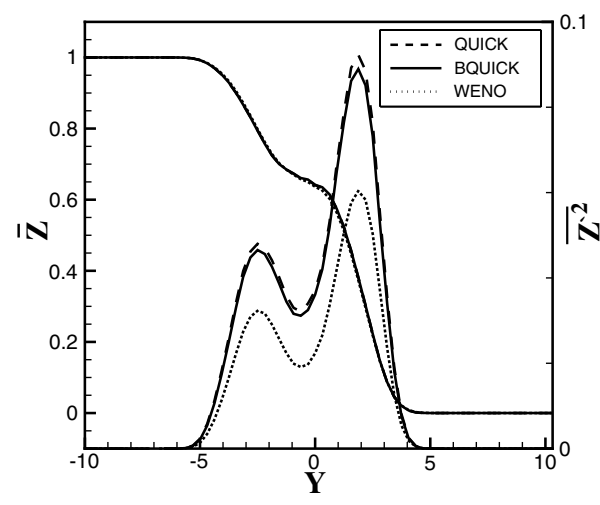

b) Time-averaged, $X=36.9$

Fig. 4 Cross-stream profile of mixture fraction and mixture fraction variance in the mixing layer. 


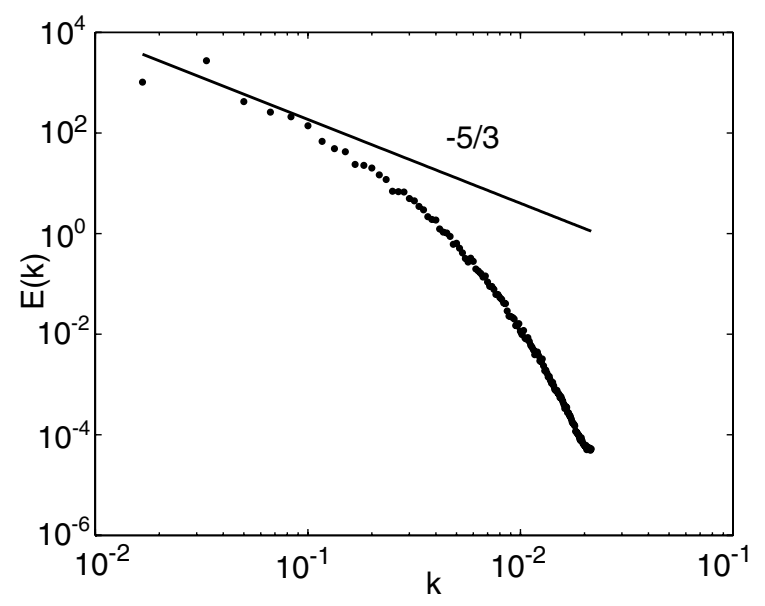

a) Streamwise velocity component

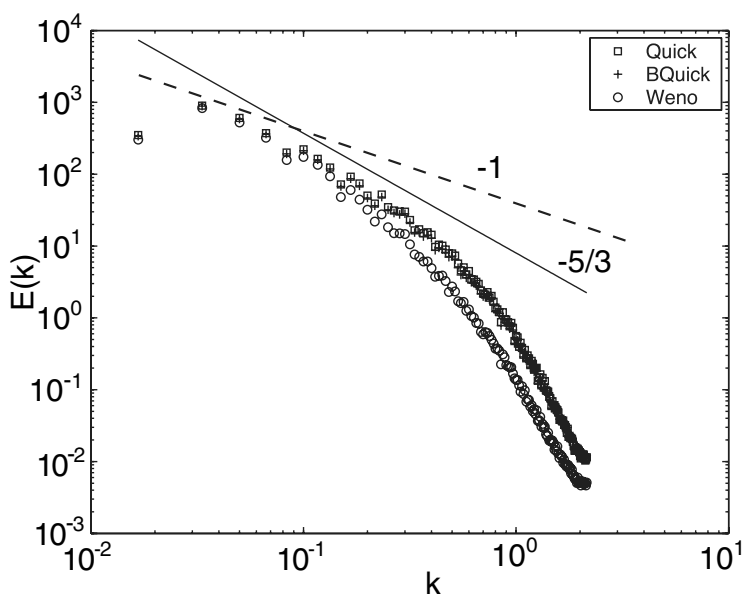

b) Mixture-fraction

Fig. 5 One-dimensional (planar averaged) streamwise energy spectrum at $Y=0$ and $t=92$.

small fraction of the computational domain and hence the variance is not significantly damped by dissipation errors. On the other hand, the mixture-fraction variance computed by the WENO scheme exhibit significantly lower levels than the BQUICK scheme: the variance is damped by about $20 \%$ compared with the QUICK/BQUICK values. All three schemes show nearly identical profiles of time-averaged mixture-fraction. However, this does not imply that the timeaveraged values of reactive scalars, like for example temperature, are identical in the three schemes. This is due to the fact that the mapping between instantaneous mixture-fraction and reactive scalars is nonlinear and the time-averaged reactive scalar values are therefore functions not only of the time-averaged mixture-fraction, but also its variance. Thus, an undamped prediction of the mixture-fraction variance is crucial.

\section{Temporal Mixing Layer}

The computational domain used for the simulation of the temporal mixing layer is initially divided into two streams separated by an interface located at $Y=0$. The initial mean velocity distribution is given by a hyperbolic tangent velocity profile. Three-dimensional perturbations with wavelengths of up to a quarter of the domain length are superimposed on the mean velocity profile. The different variables are nondimensionalized using the initial vorticity thickness $\delta_{\omega}$ and the mean velocity difference between the two streams. Based on these definitions, the initial Reynolds number is $R e_{\omega, 0}=435$. The simulation is performed on a grid with $256^{3}$ control volumes. Figure 5 a shows the one-dimensional streamwise energy spectrum of the streamwise velocity component at $t=92$. For reference, the

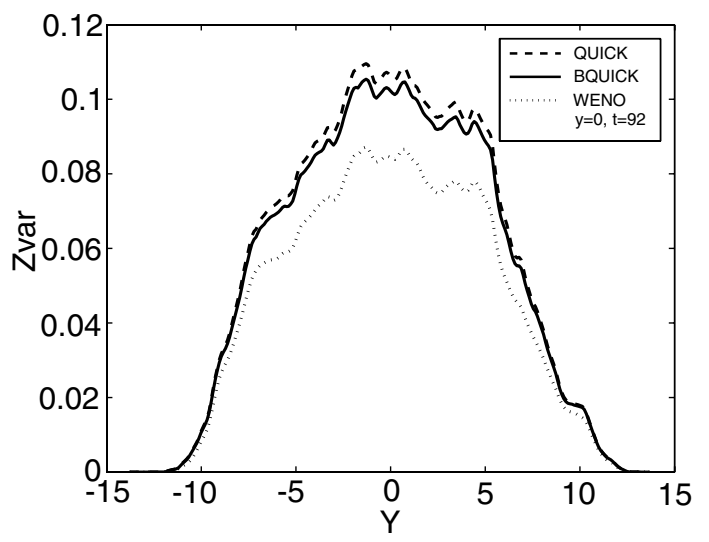

Fig. 6 Plane averaged cross-stream profiles of mixture-fraction variance obtained in the mixing layer. slope of the inertial range is indicated. The energy spectrum is in good agreement with previous work [15].

To better isolate the effect of numerical dissipation by the different schemes, the scalar transport equation is solved without molecular diffusion, thus setting the Schmidt number to infinity. In such a configuration, the energy cascade still occurs but the energy should not be dissipated at the lowest length scales. However, numerical dissipation introduced by the schemes will prevent the energy from piling up at the smallest length scales. Figure $\underline{5 b}$ shows the onedimensional energy spectra of the scalars for the three different schemes. All three schemes show a region of constant slope decay, reminiscent of an inertial range, although the slope is closer to $-5 / 3$ than to the theoretical Batchelor scaling value of -1 for infinite Schmidt number scalars. The reason for this is twofold. For one, the used Reynolds number is not high enough to sustain an extended inertial range. For another, and more important, the inherent numerical dissipation in all three schemes lowers the effective Schmidt number significantly, thereby giving results closer to the $-5 / 3$ scaling of low Schmidt number scalars. Although neither of the three schemes performs well in this DNS setting, given the constraints on the schemes, the QUICK and BQUICK schemes clearly outperform the WENO scheme, and QUICK and BQUICK yield virtually indistinguishable results.

This result can be further ascertained by considering the variance of the temporal fluctuations of the scalar (Fig. 6). The upwind correction of the BQUICK scheme has little effect on the mixturefraction variance because, once again, the fraction of computational domain where the first-order correction is applied is very small. On the other hand, the WENO profile shows lower variance values by about $15 \%$ as compared with the QUICK/BQUICK profiles.

Because no molecular diffusion is present in the case analyzed here, the turbulent velocity field should simply corrugate any arbitrary defined mixture-fraction isosurface $0<Z_{1}<1$ and the isosurface averaged gradient of the mixture-fraction should be constant in time. Figure 7 shows the nondimensional surface area $A_{Z_{1}}(t) / A_{Z_{1}}(t=0)$ of both the $Z_{1}=0.5$ isosurface and the $Z_{1}=$ 0.055 isosurface, which corresponds to the stoichiometric isosurface for methane/air combustion. In both cases, the QUICK and BQUICK results are similar, although the BQUICK scheme predicts a slightly smaller surface area for the $Z_{1}=0.055$ isosurface than the QUICK scheme. This is due to the effect of the first-order correction term acting on the $Z=0$ boundary. However, the WENO scheme shows markedly smaller surface areas than both the QUICK and the BQUICK scheme. Figure $\underline{8}$ depicts the averaged mixture-fraction gradient, nondimensionalized by $1 / \Delta x$ and conditioned on the $Z_{1}$ isosurface. An ideal, nondissipative scheme should maintain a nondimensional gradient of unity. Because of the numerical dissipation present in all three schemes, the gradient decreases over time. Whereas the BQUICK and QUICK results are similar for the 


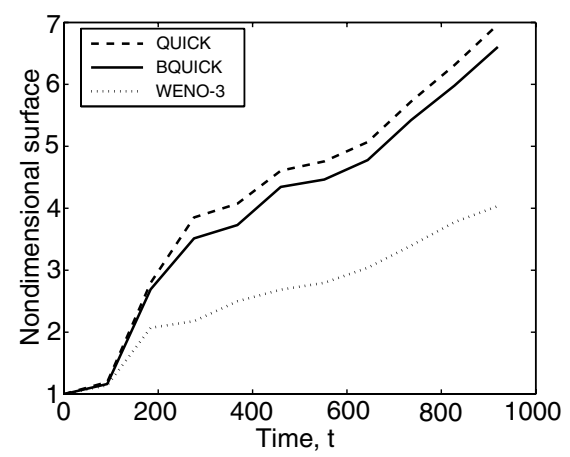

a) $\mathrm{Z}_{\mathbf{1}}=\mathbf{0 . 0 5 5}$

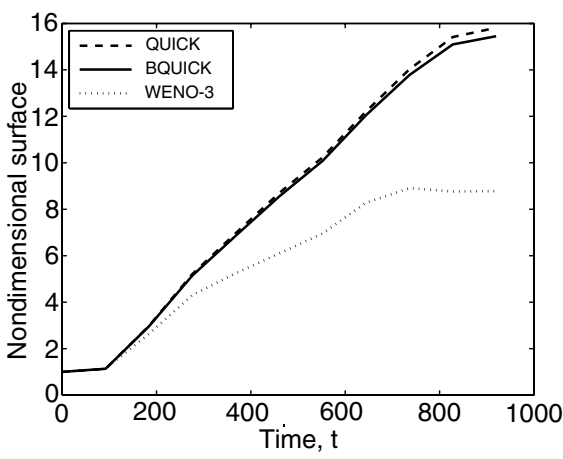

b) $\mathrm{Z}_{1}=\mathbf{0 . 5}$

Fig. 7 Temporal evolution of the $Z_{1}$ isosurface area.

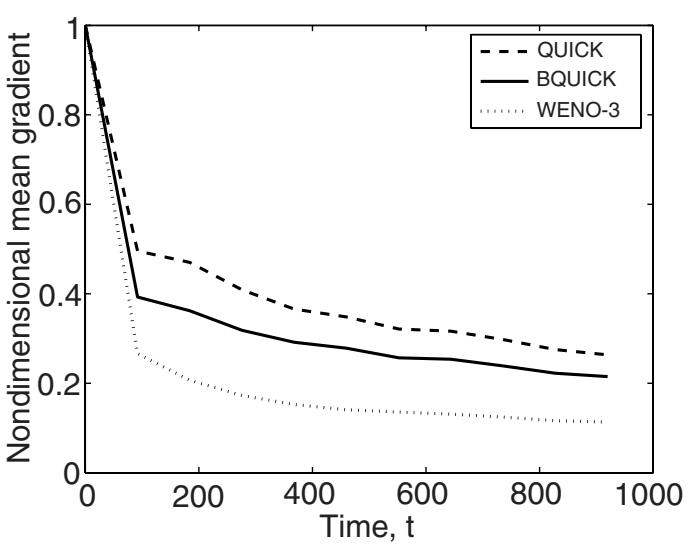

a) $Z_{1}=\mathbf{0 . 0 5 5}$

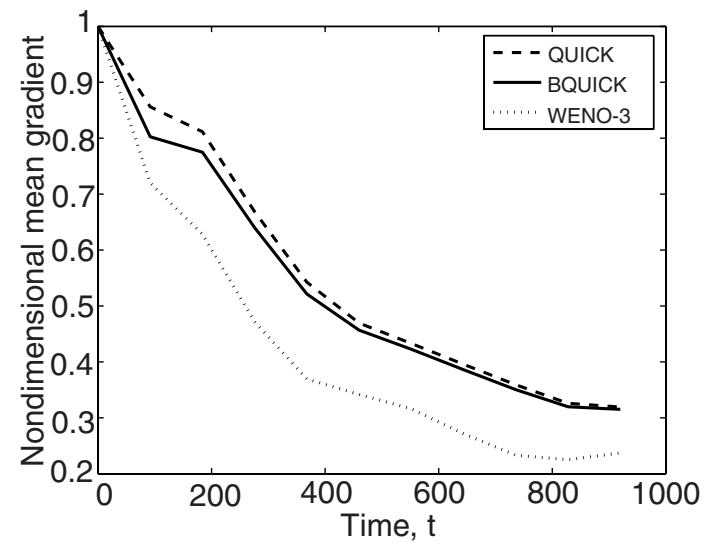

b) $\mathrm{Z}_{1}=0.5$

Fig. 8 Temporal evolution of the nondimensional mean $Z$-gradient at the $Z_{1}$ isosurface.

$Z_{1}=0.5$ isosurface, BQUICK shows gradients about $20 \%$ smaller than the QUICK scheme at $Z=0.055$ where the first-order correction at the $Z=0$ bound are active. The BQUICK gradients are still almost twice the value given by the WENO scheme in both cases and thus are significantly better. Note that these results are consistent with the results of the one-dimensional test case presented in the preceding section.

Finally, Fig. $\underline{9}$ shows the $Z_{1}=0.055$ isosurface for both the BQUICK and the WENO scheme. It is seen that WENO does not show the small structures as the BQUICK scheme does. As a consequence, the WENO scheme is not able to capture small pockets of low mixture-fraction surrounded by high mixture-fraction and vice versa, and the isosurface area is significantly smaller, cf. Fig. 7.

Also, note that in typical technical combustion cases, the stoichiometric mixture-fraction is small, for example it is $Z_{\mathrm{st}}=$ 0.055 in the case of methane/air combustion. This leads to very strong density gradients in the mixture-fraction space close to $Z=0$. Because the QUICK scheme leads to extended excursions below zero, large unphysical temporal changes in density can occur. In the incompressible case, this results in ill-conditioned Poisson equations
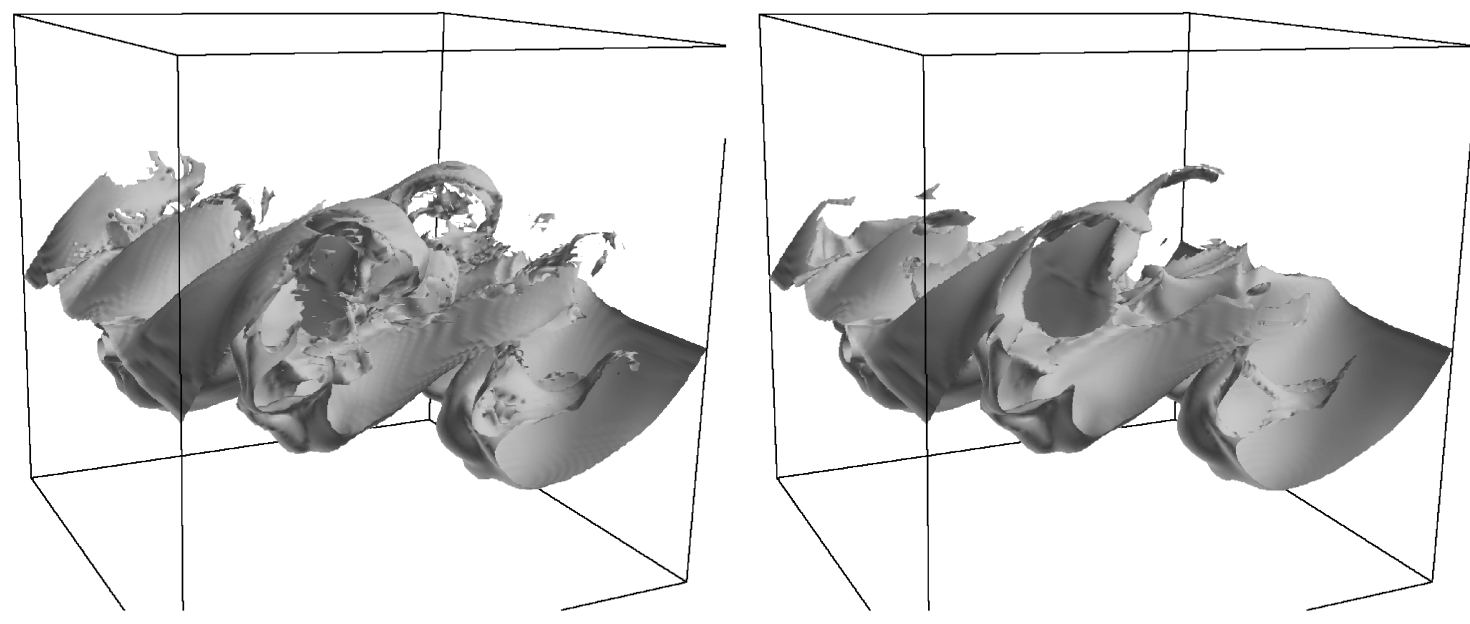

a) BQUICK

b) WENO

Fig. $9 Z_{1}=0.055$ isosurface at $t=46$. 
leading to algorithmic instability. Because the BQUICK scheme maintains the physical bounds, these algorithmic instabilities are not observed.

\section{Conclusions}

A modification to the QUICK scheme has been presented that ensures scalar boundedness. This bounded QUICK scheme shows accuracy comparable to the original QUICK scheme and is superior in performance to a standard third-order WENO scheme, while still of comparable numerical cost. The BQUICK scheme is geared towards LES of industrially relevant reacting flows in complex geometries. BQUICK is applicable to both passive and reactive scalars and thus to a wide range of turbulent combustion models. Numerical tests show that the BQUICK scheme preserves the variance of the temporal fluctuations of scalar quantities better than the WENO scheme in the case of a spatial mixing layer. Also, the BQUICK scheme preserves the mean scalar gradient conditioned on a scalar isosurface significantly better than the WENO scheme. Both properties are essential in predicting the turbulent combustion process correctly, indicating that the BQUICK scheme is a superior scheme for LES of turbulent reacting flows.

\section{Acknowledgments}

This work was carried out while V. Raman was a Research Associate at the Center for Turbulence Research. The authors would like to thank Frank Ham, Magnus Svard, and Heinz Pitsch for fruitful discussions.

\section{References}

[1] Ham, F., Apte, S., Iaccarino, G., Wu, X., Herrmann, M., Constantinescu, G., Mahesh, K., and Moin, P., "Unstructured LES of Reacting Multiphase Flows in Realistic Gas Turbine Combustors," Annual Research Briefs 2003, Center for Turbulence Research, Stanford, CA, 2003, pp. 139-160.

[2] di Mare, F., Jones, W. P., and Menzies, K. R., "Large Eddy Simulation of a Model Gas Turbine Combustor," Combustion and Flame, Vol. 137, No. 3, 2004, pp. 278-294.

[3] Balsara, D. S., and Shu, C.-W., "Monotonicity Preserving Weighted
Essentially Non-Oscillatory Schemes with Increasingly High Order of Accuracy," Journal of Computational Physics, Vol. 160, No. 2, 2000, pp. 405-452.

[4] Morinishi, Y., Lund, T. S., Vasilyev, O. V., and Moin, P., "Fully Conservative Higher Order Finite Difference Schemes for Incompressible Flow," Journal of Computational Physics, Vol. 143, No. 1, 1998, pp. $90-124$.

[5] Peters, N., Turbulent Combustion, Cambridge Univ. Press, Cambridge, UK, 2000.

[6] Pierce, C. D., "Progress-Variable Approach for Large-Eddy Simulation of Turbulence Combustion," Ph.D. Thesis, Stanford Univ., Stanford, CA, 2001.

[7] Zalesak, S. T., "Fully Multidimensional Flux-Corrected Transport Algorithms for Fluids," Journal of Computational Physics, Vol. 31, No. 3, 1979, pp. 335-362.

[8] Jiang, G.-S., and Peng, D., "Weighted ENO Schemes for HamiltonJacobi Equations," SIAM Journal on Scientific Computing, Vol. 21, No. 6, 2000, pp. 2126-2143.

[9] Moureau, V., Lartigue, G., Sommerer, Y., Angelberger, C., Colin, O., and Poinsot, T., "Numerical Methods for Unsteady Compressible Multi-Component Reacting Flows on Fixed and Moving Grids," Journal of Computational Physics, Vol. 202, No. 2, 2005, pp. 710-736.

[10] Kennedy, C. A., and Carpenter, M. H., "Several New Numerical Methods for Compressible Shear-Layer Simulations," Applied Numerical Mathematics, Vol. 14, No. 4, 1994, pp. 397-433.

[11] Leonard, B. P., "Stable and Accurate Convective Modelling Procedure Based on Quadratic Upstream Interpolation," Computer Methods in Applied Mechanics and Engineering, Vol. 19, No. 1, 1979, pp. 59-98.

[12] Pierce, C. D., and Moin, P., "Progress-Variable Approach for LargeEddy Simulation of Non-Premixed Turbulent Combustion," Journal of Fluid Mechanics, Vol. 504, 2004, pp. 73-97.

[13] Brown, P. N., Hindmarsh, A. C., and Petzold, L. R., "Consistent Initial Condition Calculation for Differential-Algebraic Systems," SIAM Journal on Scientific Computing, Vol. 19, No. 5, 1998, pp. 1495-1512.

[14] Jiang, G. S., and Shu, C. W., "Efficient Implementation of Weighted ENO Schemes," Journal of Computational Physics, Vol. 126, No. 1, 1996, pp. 202-228.

[15] Vreman, B., Geurts, B., and Kuerten, H., "A Priori Tests of Large Eddy Simulation of the Compressible Plane Mixing Layer," Journal of Engineering Mathematics, Vol. 29, No. 4, 1995, pp. 299-327.

C. Kaplan

Associate Editor 